\title{
Skin collagen defects in a patient with juvenile hyaline fibromatosis
}

\author{
Barbara Lubec, I Steinert, F Breier, W Jurecka, K Pillwein, S Fang-Kircher
}

\begin{abstract}
Juvenile hyaline fibromatosis is a rare disorder characterised by multiple subcutaneous tumours, gum hypertrophy, muscle weakness, and flexion contractures of the large joints. Histology shows an abundance of a homogenous, amorphous, acidophilic extracellular matrix in which spindle shaped cells are embedded forming minute streaks. It has been previously suggested that collagen abnormalities may be involved. A 14 month old girl with this syndrome is described in whom postmortem western blot studies were performed. These studies revealed an absent pro- $\alpha 2$ (I) chain and an absent collagen type III chain in skin but not in the other organs examined.
\end{abstract}

(Arch Dis Child 1995; 73: 246-248)

Keywords: juvenile hyaline fibromatosis, collagens, collagen type III.

Juvenile hyaline fibromatosis (McKusick 228600, including puretic syndrome, Murray's syndrome, or systemic juvenile fibromatosis) is a rare disorder characterised by multiple subcutaneous tumours, gum hypertrophy, muscle weakness, and flexion contractures of the large joints. The skin tumours emerge around 2 years of age as pink papules. They grow slowly, cause deformities of the face, neck and scalp, and recur after surgical removal. Patients also suffer from recurrent infections, which often lead to death in early childhood. Skeletal osteolytic and osteoplastic lesions have been described. The aetiology of this autosomal recessive disorder is still unknown, but its predominant manifestations at sites with increased mechanical strain suggest that it might be due to a defective metabolism of collagen fibrils. Histological studies of the skin lesions demonstrated an abundance of a homogeneous, amorphous, acidophilic ground substance, in which spindle shaped 'tumour' cells are embedded, forming minute streaks.

The observation of densely, often concentrically arranged intracellular fibrils and an abundance of extracellular matrix also indicate a primary defect of collagen fibrils. Earlier studies postulated that the increased deposition of hyaline material in skin tumours of patients with juvenile hyaline fibromatosis is due to an increased synthesis of glycosaminoglycans by fibroblasts. ${ }^{1}$ By contrast, Kayashima and coworkers recently reported that immunochemical and biochemical studies on juvenile hyaline fibromatosis tumours point to an involvement of type VI collagen in this rare disorder. ${ }^{2}$

Here, we describe postmortem immunochemical studies on collagens by western blotting in several organs of a child with juvenile hyaline fibromatosis revealing deficient skin collagen.

\section{Case report}

A girl was born in 1992 to unrelated Austrian parents; she had a healthy 10 year old sister. One son of the mother's sister suffered from hypertrophic gingiva; a cousin had trisomy 21 . In some members of the mother's family (no details known) contractures of several fingers were observed as individual symptoms.

During pregnancy increased plasma $\alpha$-fetoprotein concentrations were observed but no signs of a neural tube defect were found. Because of premature rupture of membranes in the 26th gestational week and signs of intrauterine infection the baby girl was delivered by caesarean section in the 28 th week of gestation. Apgar scores were $7 / 8 / 8$ at 1,5 , and 10 minutes; the child weighed $1390 \mathrm{~g}$ and was $38 \mathrm{~cm}$ long. After some feeding difficulties she developed normally during the first months of life.

During the neonatal period the parents detected contractures of the left elbow and of the fourth finger of the left hand. Subsequently, livid papules on the skin covering all large joints, hyperplastic gingivae and papillomas in the anal and perigenital area appeared (fig 1) and the child was hospitalised at the age of 11 months. According to the child's paediatrician who suspected juvenile hyaline fibromatosis the clinical diagnosis was confirmed and established by skin biopsy.

For electron microscopy tissue from the skin biopsy specimen was fixed in $2.5 \%$

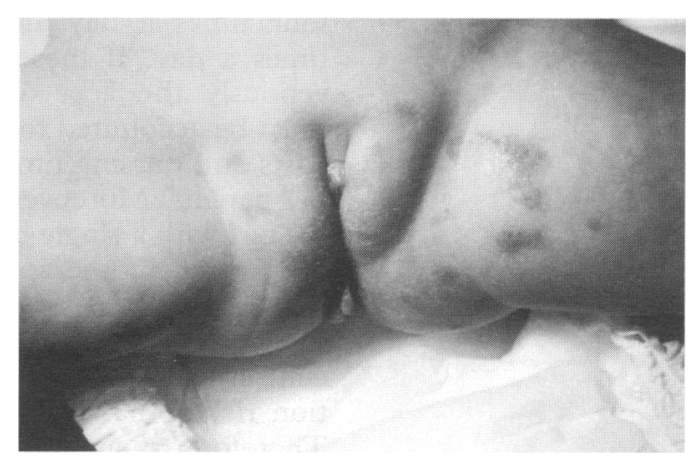

Figure 1 Plaque-like, reddish infiltrations with perigenital localisation and central whitish nodules (representing miliar cysts); perianal papillomatous reddish lesions could be observed.
obsts), 




glutaraldehyde and $0 \cdot 1 \mathrm{M}$ phosphate buffer, postfixed in osmium tetroxide and uranyl acetate, and embedded in Epon 812. Ultrathin sections were stained with lead citrate and uranyl acetate and examined under a Jeol lolo transmission electron microscope.

Low power magnification showed a tumorous proliferation of numerous fibroblast-like cells with a sharply demarcated border, forming pseudopodian like protrusions. The nucleus revealed invaginations surrounded by a fine granular electron dense material. In the cytoplasm numerous membrane bound vesicles containing a fine fibrillar material could be detected. These cells were embedded in a profuse stroma. Similarly, the stroma was composed of densely packed delicate microfibrils, which were arranged diffusely or in parallel resembling the material seen intracellularly (fig 2). These findings are fully compatible with the diagnosis. ${ }^{34}$

At that time routine biochemical tests were within the normal range, except $\alpha$-fetoprotein, which was increased twofold. Hypochromatic microcytic anaemia was present. $x$ Ray examination of the skeleton showed decreased bone density and defectively positioned joints, most probably due to contractures. There were no signs of visceral involvement clinically or radiologically. Ultrasound examination of the brain showed normal anatomy but clinically a moderate psychomotor retardation was detectable.

At the age of 14 months the girl was admitted to hospital because of tracheobronchitis and bilateral pneumonia with a fulminant course; she died two days later.

At necropsy no signs of visceral involvement could be found.

\section{Experimental}

The affected organs - skin, gum tissue, kidney, heart, and liver - were obtained at necropsy, minced to small pieces and washed in isotonic saline, homogenised in an ice bath, and acid salt soluble collagen was extracted. Homogenised material was incubated in $0.05 \mathrm{M}$ acetic acid containing 0.005 $\mathrm{M}$ EDTA and $5 \mathrm{mg}$ pepsin (Merck)/100 mg (wet weight) of tissue; the incubation took place for 72 hours at $4^{\circ} \mathrm{C}$ on a shaker. Subsequently the samples were spun down in a refrigerated centrifuge at $4000 \times g$, the supernatant filtered through Millipore filters, and the protein concentration evaluated by the method of Bradford using a commercially available colorimetric assay (Bio-Rad).

Sodium dodecylsulphate-polyacrylamide gel electrophoresis (SDS-PAGE) was carried out according to the method of Laemmli. ${ }^{5}$ One hundred micrograms of protein were applied per well and gels were stained with a commercially available silver stain kit (Bio-Rad) and corresponding duplicates were unstained and transferred to nylon membranes for western blotting. Western blots were carried out according to a standard method as given in a previous paper. ${ }^{6}$ Antibodies against type I, type III, type IV, and type VI collagen (Institute Pasteur, Paris) were used.

\section{QUANTIFICATION OF SKIN COLLAGEN}

After mechanical removal of the subcutaneous tissue, skin was defatted by diethylether/ chloroform (1/1) and freeze dried. The dry weight was taken and 4-hydroxyproline was determined by the method after Woessner. ${ }^{7}$ The hydroxyproline content/mg skin was calculated and considered to reflect total collagen content of the cutis.

The same organs (except gum tissue) of five age matched children were used for comparison of gels, and skin of five age matched children was used for quantitative collagen studies.

\section{Results}

SKIN COLLAGEN CHANGES

The results of the qualitative studies (electrophoresis and western blots) are shown in figs 3 and 4. The SDS-PAGE electrophoresis showed the absence of the pro- $\alpha 2(\mathrm{I})$ band as well as the absence of the band in the position of the collagen (III) chain in our patient. Western blots using anticollagen (I) revealed the presence of a single band; development with antibodies against collagen (III) showed the absence of immunoreactive collagen (III). The other antibodies (against types IV, VI) applied showed normal patterns compared with the five control specimens.

The high molecular weight bands representing cross linked collagens, that is polymers, did not show any differences in comparison with the control group.

\section{QUANTITATIVE STUDIES}

Mean (SD) values of 4-hydroxyproline/mg skin of the patient (triplicates) were 
Figure 3 Western blot pattern of collagen type III. From left: lane I: the band representing collagen type III in kidney extract of our patient; lane II: the band representing collagen type III in skin of a healthy control; lanes III-VI: no immunoreactive band for collagen type III is seen applying skin collagen extracts of the patient; and lane VII: collagen type III in the heart collagen extract of our patient.

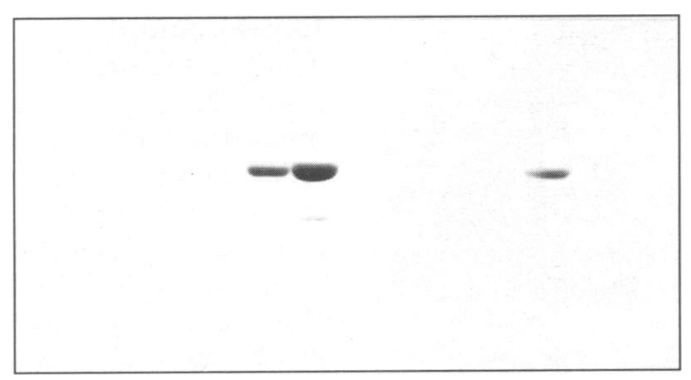

comparable with those in the control group ( 86 (2.3) $\mu \mathrm{g} / \mathrm{mg}$ skin in triplicates of the patient and $79(11.9) \mu \mathrm{g} / \mathrm{mg}$ skin in the controls). SDS-PAGE and western blotting of the other organs failed to show any qualitative or (semi)quantitative changes. The examined parts of the organs gum, kidney, heart, and liver did not show any morphological changes.

\section{Discussion}

The collagen defects found in our patient have been never reported before and show a complex pattern. In 1987 Sasaki and coworkers described an Ehlers-Danlos syndrome variant characterised by the deficiency of the pro- $\alpha 2$ chain of type I procollagen in skin. ${ }^{8}$ The clinical manifestation, however, was totally different from our observation as the patient reported presented with cardiovascular abnormalities, hypermobility of joints, and hyperextensibility of the skin. The deficiency of skin collagen pro- $\alpha 2$ chain has been reported before in other clinical conditions, for example, osteogenesis imperfecta and Marfan's syndrome. ${ }^{9}$

Pihlajaniemi and coworkers reported on the cloning of a procollagen pro- $\alpha 2(\mathrm{I})$ collagen gene with a frameshift mutation in skin fibroblasts of a patient with osteogenesis imperfecta ${ }^{10}$; this paper shed new light on the complexity of collagen formation and metabolism. The mutation described prevented the incorporation of pro- $\alpha 2$ (I) chains into the normal type I procollagen heterotrimer resulting in secretion of only pro$\alpha 1$ (I) chains. Other mechanisms for pro- $\alpha 2(\mathrm{I})$ pathologies have been described in addition: several forms of osteogenesis analysed at the protein level are caused by deletions in the collagenous region and/or decreased synthesis of the procollagen chains. ${ }^{11-16}$ One observation in

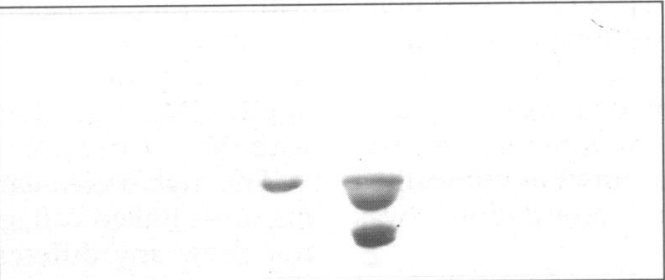

Figure 4 Western blot revealing the collagen type I pattern (overload on purpose to show that even at high concentrations no $\alpha 2$ band was visible). Left lane: one single band in the collagen skin extract of our patient representing the $\alpha 1$ (I) chain and right lane: a representative pattern as seen in a normal collagen skin extract showing the $\alpha 1(I)$ chain above and the $\alpha 2(I)$ chain below.
Marfan's syndrome showed an insertion in the pro- $\alpha 2$ (I) collagen domain and one observation in Ehlers-Danlos syndrome type VII had a defect preventing the cleavage of the pro- $\alpha 2(\mathrm{I})$ amino propeptides. ${ }^{17} 18$

Kratochwil and coworkers showed the retrovirus induced insertional mutation in mov13 mice affecting collagen I expression in a tissue specific manner. ${ }^{19}$ This model could explain our findings of tissue specificity (skin) and chain specificity (only $\alpha 2$ chain affected).

The cited mechanisms are able to explain the findings of the deficient type III chains as well. It is interesting that these findings of collagen abnormalities are compatible with life and present with entirely different clinical features. It is also of major interest that no distinct cross linking disturbances were found in our patient as revealed by apparently and semiquantitatively normal bands in the high molecular weight region of polyacrylamide gel electrophoresis.

1 Finlay AY, Ferguson SD, Holt PJA. Juvenile hyaline fibromatosis. Br F Dermatol 1983; 108: 609-16.

2 Kayashima KI, Katagiri K, Shinkai H, Ono T. Is juvenile hyaline fibromatosis a disease of type VI collagenosis? In: Ishibashi Y, Nakagawa H, Suzuki H, eds. Electron microscopy in dermatology - basic and clinical research. Amsterdam: Elsevier Science BV, 1994: 329-34.

3 Kitano Y, Horiki M, Aoki T, Sagami S. Two cases of juvenile hyaline fibromatosis: some histological, juven microscopical and tissue culture observations. Arch Dermatol 1972; 106: 877-83.

4 Woyke S, Domagala W, Olszewski W. Ultrastructure of fibromatosis multiplex juvenilis. Cancer 1970; 26: $1157-68$.

5 Laemmli UK. A cleavage of structural proteins during the assembly of the head of bacteriophage T4. Nature 1970;

6 Lubec B, Weninger M, Popow C, Vierhapper H, Lunec J, Lubec $\mathrm{G}$. In vivo monitoring of serum protein cross linking in patients with diabetes mellitus. Amino Acids 1993; 4: $111-9$.

7 Woessner JF. Determination of hydroxyproline in tissue and protein sample containing small proportions of this amino acid. Arch Biochem Biophys 1961; 93: 440-7.

8 Sasaki T, Arai K, Ono M, Yamaguchi T, Furuta S, Nagai Y. Ehlers Danlos syndrome. A variant characterized by the deficiency of pro alpha 2 chain of type I procollagen. Arch Dermatol 1987; 23: 76-9.

9 Prockop DJ, Kivirikko KI. Collagen and its disorders. N Engl f Med 1984; 311: 376-86.

10 Pihlajaniemi T, Dickson LA, Pope FM, et al. Osteogenesis imperfecta: cloning of a pro- $\alpha 2(\mathrm{I})$ collagen gene with 12941-4.

11 Barsh GS, Byers PH. Reduced secretion of structurally abnormal type I procollagen in a form of osteogenesis imperfecta. Proc Natl Acad Sci (USA) 1981; 78: 5142-6.

12 Barsh GS, David KE, Byers PH. Type I osteogenesis imperfecta: a nonfunctional allele for pro alpha(I) chains of type I procollagen. Proc Natl Acad Sci (USA) 1982; 79: 3838-42.

13 Byers PH, Shapiro JR, Rowe DW, David KE, Holbrook KA. AbnormAl alpha 2 chain in type I collagen from a patient with a form of osteogenesis imperfecta. 7 Clin Invest 1983; 71: 689-97.

14 Williams CJ, Prockop DJ. Synthesis and processing of a type I procollagen containing shortened pro alphal (I) chain by fibroblasts from a patient with osteogenesis imperfecta. fibroblasts from a patient with

15 de Wet WJ, Pihlajaniemi T, Myers JC, Kelly TE, Prockop DJ. Synthesis of a shortened pro alpha2(I) chain and DJ. Synthesis of a shortened pro alpha2(In chain and
decreased synthesis of pro alpha2(I) chains in a proband decreased synthesis of pro alpha2(I) chains in a proband with ost

16 Sippola M, Prockop DI. A shortened pro alpha 2(I) chain in a mild variant of osteogenesis imperfecta. $\mathcal{F}$ Cell Biol 1983;

17 Byers PH, Siegel R, Peterson KE, et al. Marfan syndrome: abnormal alpha2 chain in type I collagen. Proc Natl Acad Sci (USA) 1981; 78: 7745-9.

18 Steinman B, Tuderman L, Peltonen L, Martin GR, McKusick VSA, Prockop DJ. Evidence for a structural mutation of procollagen type I in a patient with the Ehlers Danlos syndrome type VII. $\mathcal{F}$ Biol Chem 1980; 225: 8887-93.

19 Kratochwil K, von der Mark K, Kollar EJ, et al. Retrovirus induced insertional mutation in mov 13 mice affects collagen I expression in a tissue specific manner. Cell 1989; 57 807-16. 